\title{
Article
}

\author{
Doi 10.5943/sif/6/1/40
}

\section{New record of luminescent Mycena chlorophos (Mycenaceae) from Western Ghats of India}

\section{Arya CP, Ratheesh S and Pradeep CK*}

Microbiology Division, Jawaharlal Nehru Tropical Botanic Garden \& Research Institute, Palode, Thiruvananthapuram, 695 562, Kerala, India

Arya CP, Ratheesh S, Pradeep CK 2021 - New record of luminescent Mycena chlorophos from Western Ghats of India. Studies in Fungi 6(1), 507-513, Doi 10.5943/sif/6/1/40

\begin{abstract}
An interesting luminescent Mycena was collected from dead bamboo culms on several occasions from an evergreen forest in Kerala State, India. Detailed morphological and molecular studies with nrITS sequence data confirmed it as Mycena chlorophos. A reappraisal of the species along with comprehensive description, photographs and a discussion with related species is provided. This forms the first record of this species from India.
\end{abstract}

Keywords - Bioluminescent - new record - nrITS - Omphalotaceae - Pleurotaceae Physalacriaceae

\section{Introduction}

Bioluminescent organisms constitute an important component in the living world and spread over in 700 genera (Herring 1987). Of these, luminescent fungi form a major part with 98 taxa (Cortés-Pérez et al. 2019). Most of the bioluminescent fungi belong to the families Mycenaceae, Omphalotaceae Pleurotaceae, and Physalacriaceae with Mycenaceae as the most species rich with 69 taxa (Desjardin et al. 2008, Cortés-Pérez et al. 2019). The luminosity varies with species as in some cases only the mycelium is luminescent whereas in some species both mycelium and basidiomata are luminescent. In a few species, only basidiomata have been reported as luminescent while the mycelium is non-luminescent (Cortés-Pérez et al. 2019).

The knowledge on Indian luminescent fungi is scanty, sporadic and incomplete (Vrinda et al. 1999, Kumar \& Kaviyarasan 2012, Aravindakshan et al. 2012, Karunarathna et al. 2020). During our inventory studies on the agaricoid basidiomyetes of Kerala State, India, we came across a luminescent Mycena which glows on dark with a pale green light. Detailed morphological and molecular study revealed it as M. chlorophos (Berk. \& M.A. Curtis) Sacc. which is currently recognized as pantropical however, has not been reported earlier from India. Here we describe and discuss this agaric comprehensively with some additional notes.

\section{Materials \& methods}

\section{Morphological studies}

Mycena basidiomata were collected during the monsoon months of 2019-2020, in tropical evergreen forests of Jawaharlal Nehru Tropical Botanic Garden and Research Institute (JNTBGRI) campus (150 $\mathrm{m}$ a.s.1.). Descriptions were derived from recording the morpho-anatomical features of fresh samples, and the color codes follow Kornerup \& Wanscher (1978). Microscopic features were 
examined from sections made from dried specimens, measured in 3\% aqueous $\mathrm{KOH}$ and stained with $1 \%$ Congo red. Thirty basidiospores were measured for length and width. Basidiospore measurements include both the mean and the standard deviation for both the length and the width, together with the range of spore quotient $(\mathrm{Q}$, length/width ratio) and its mean value $(\mathrm{Qm})$. Basidiospore measurements exclude the hilar appendix. All materials examined are deposited at the Mycological Herbarium of Jawaharlal Nehru Tropical Botanic Garden and Research Institute, Thiruvananthapuram [TBGT (M)]. Facesoffungi number was registered and added as per Jayasiri et al. (2015).

\section{DNA sequencing and phylogenetic analysis}

Genomic DNA was extracted from fresh specimens of Mycena chlorophos collected from Kerala, India following protocols in Izumitsu et al. (2012). The nuclear ribosomal Internal Transcribed Spacer region (nrITS) was amplified and sequenced from the specimens of Mycena chlorophos. PCR reactions were performed with the primer pair ITS1 and ITS4 (White et. al. 1990). The protocols for PCR amplification and sequencing followed Kumar et al. (2018b). The newly generated sequences were deposited in GenBank (MZ389977).

The molecular phylogenetic analysis was performed using nrITS sequences newly generated from the Kerala material and those retrieved from GenBank. The nrITS sequence of Mycena chlorophos (515bp) along with those retrieved from GenBank were aligned using MAFFT web tool (www.ebi.ac.uk/Tools/msa/mafft/) with default settings. The final aligned data matrix of nrITS sequences from 29 taxa, including two species of Hemimycena as outgroups, were then imported into BioEdit v7.2.6.1 (Hall 1999) for manual adjustment. Hemimycena ochrogaleata (J. Favre) M.M. Moser and Hemimycena tortuosa (P.D. Orton) Redhead were selected as outgroup following Chang et al. (2020). Maximum likelihood (ML) analysis was performed in the web platform http://iqtree.cibiv.univie.ac.at (Trifinopoulos et al. 2016) with 1000 ultrafast bootstrap replicates. Transition model with equal frequency (TIM2+F+G4) was selected automatically as the best fit substitution model as per BIC score in the same web platform. The aligned sequence dataset has been deposited in TreeBase (ID: S28533). The phylogram inferred from ML analysis is displayed with MEGA X (Kumar et al. 2018a).

\section{Results}

\section{Taxonomy}

Mycena chlorophos (Berk. \& M.A. Curtis) Sacc., Syll. Fung. (Abellini) 5: 301 (1887) Figs 1-2 Index Fungorum number: IF147895; Facesof fungi number: FoF10625

Pileus 3-25 mm diam., parabolic to spherical in buds, becoming convex to plano-convex with a shallow depression at centre when mature; surface dark blonde to grayish brown (5D4/6F6/6F8/8D3) uniformly in buds becoming pure white with grayish or brownish disc when mature, pellucid striate, viscid with a thick gluten cover in wet weather, glabrous, shiny; disc become oak brown to mustard brown (4D6/5E4-5E6) in dry weather with paler margin, striate, sticky; margin straight, crenate. Lamellae adnexed, (rarely with a pseudocollarium) white, up to 4 mm wide, close with lamellulae of 1-3 lengths; edge concolorous to the sides, entire. Stipe 5-20 $\times$ 0.5-20 mm, central, terete, cylindric, curved, equal arising from a cupulate basal disc, narrowly hollow; surface transparent white except at the extreme base which is pale grayish, smooth, glabrous, non-viscid. Context white, thin, up to $1 \mathrm{~mm}$ thick. Odor mild, not characteristic. Spore print off white to cream.

Luminescence - Pileus and hymenophore emitting bright, greenish light in the dark; luminescence not observed in other parts of the basidiomata. Basidiospores $6-10 \times 4-6.8 \mu \mathrm{m}\left(\mathrm{L}^{\mathrm{m}}=\right.$ 7.72, $\left.\mathrm{W}^{\mathrm{m}}=5.66\right) ; \mathrm{Q}=1.06-1.69 ; \mathrm{Qm}=1.36$, ellipsoid, smooth, amyloid, thin-walled, hyaline. 

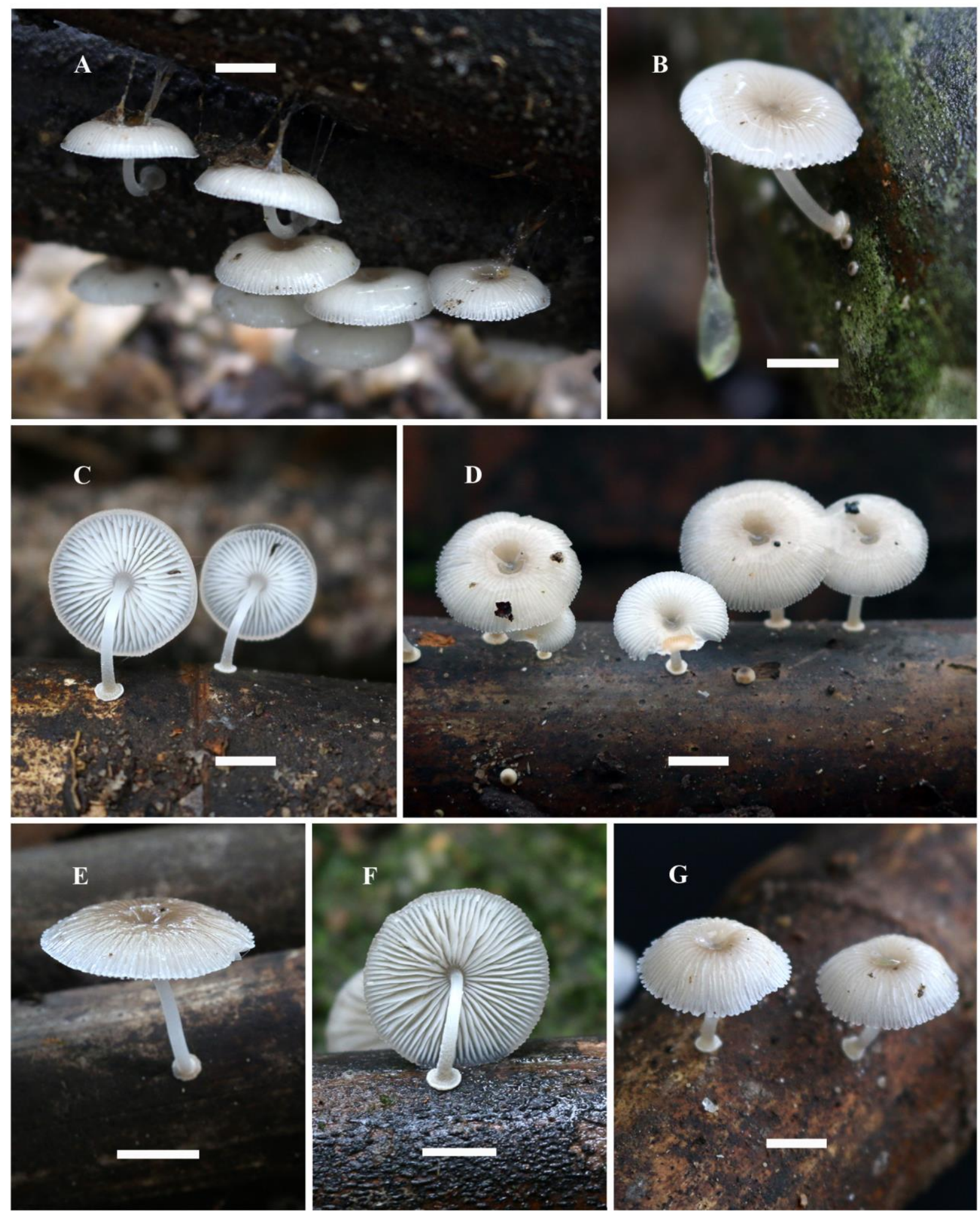

Fig. 1 - Mycena chlorophos. A-G Habit in situ. B Basidioma with gluten oozing. C Lamella view. E, G Basidioma in dry weather. F Basidioma with discoid stipe base. Scale bars $=10 \mathrm{~mm}$.

Basidia are of two types: normal basidia less abundant, 26-36 × 6-8 $\mu \mathrm{m}$, 4-sterigmate, clavate, thin-walled, hyaline; short, subglobose to vesiculose clavate basidia $16.8-22 \times 12-16 \mu \mathrm{m}$, abundant, 4-sterigmate, sterigmata up to $13 \mu \mathrm{m}$ long. Lamella edge sterile, non-gelatinised. Cheilocystidia abundant, 28-68 $\times 5.6-20 \mu \mathrm{m}$, fusoid-ventricose, narrowly to broadly fusiform, broadly lageniform, apically narrowed to a long thin, simple, whip like extension, smooth, hyaline, thin-walled. Pleurocystidia absent. Subhymenium hyphal, non-gelatinous. Hymenophoral trama 
regular composed of slightly inflated hyphae, up to $11 \mu \mathrm{m}$ diam., dextrinoid, thin-walled, nongelatinous. Pileipellis an ixotrichoderm, up to $145 \mu \mathrm{m}$ thick, composed of repent, radially arranged, widely spaced hyphae, 2.4-3.2 $\mu \mathrm{m}$ diam., cylindrical, thin, highly branched, coralloid with clavate, broadly clavate to globose terminal cells $14.4-36.8 \times 96-20.8 \mu \mathrm{m}$, densely spinulose, or with needle like out growths, embedded in a gelatinous matrix, hyaline, inamyloid, thin-walled. Pileus marginal cells abundant, 48-92 × 11-20 $\mu$ m, fusoid to fusoid-ventricose, with a long whip like apex, moderately spinulose over the central part, elsewhere smooth, hyaline, thin-walled. Hypodermium composed of inflated hyphae, up to $21 \mu \mathrm{m}$ diam., hyaline, dextrinoid, nongelatinous, thin-walled. Pileus trama composed of interwoven hyphae, 4-12 $\mu \mathrm{m}$ diam., cylindrical to inflated, hyaline, dextrinoid, nongelatinous, thin-walled. Stipitipellis a cutis of repent hyphae with scattered to clustered caulocystidia, almost similar to cheilocystidia in shape, 30-144 × 11-24 $\mu \mathrm{m}$, fusoid to fusoid-ventricose, with long whip like apices, hyaline, nongelatinous, thin-walled. Clamp connections common in all tissues.

Habitat, phenology and known distribution - Solitary to scattered on dead, decaying bamboo culms (Ochlandra tavancorica) in tropical evergreen forest in Kerala State, India. May - October. Other reports include Malaysia, Japan, Sri Lanka, Borneo, Papua New Guinea, Australia and Brazil (Desjardin et al. 2010, Chew et al. 2014).

Materials examined - India, Kerala State, Thiruvananthapuram district, Palode, JNTBGRI campus, 22 Jul 2019, TBGT(M)17871; ibid., 24 Jul 2019, TBGT(M)17887; ibid., 13 Aug 2019, TBGT(M)17926; ibid., 05 Sep 2019, TBGT(M)17957; ibid., 16 Oct 2019, TBGT(M)17993; ibid., 24 Oct 2019, TBGT(M)17999; 25 Oct 2019, TBGT(M)18002; 21 May 2020, TBGT(M)18075; 22 May 2020, TBGT(M)18078; ibid., 23 May 2020, TBGT(M) 18080; ibid., 25 May 2020, TBGT(M)18084; ibid., 01 Jun 2020, TBGT(M)18088, ibid., 04 Jun 2020, TBGT(M)18093; ibid., 05 Jun 2020, TBGT(M)18094; ibid., 08 Jun 2020, TBGT(M)18095; ibid., 09 Jun 2020, TBGT(M)18100; ibid., 10 Jun 2020, TBGT(M)18104; ibid., 08 Jul 2020, TBGT(M)18151; ibid., 09 Jul 2020, TBGT(M)18161; ibid., 03 Aug 2020, TBGT(M)18218; ibid., 05 Aug 2020, TBGT(M)18232.

\section{Discussion}

Diagnostic features of Mycena chlorophos include: small to medium sized basidiomata; white, plano-convex, pellucid striate pileus covered with separable thick gluten; adnexed, close, white lamellae with lamellulae of 1-3 lengths; stipe white, arising from a cupulate basal disc; amyloid, ellipsoid, basidiospores; sterile lamella edge with abundant fusoid ventricose-rostrate to broadly fusiform cheilocystidia with long narrow whip like apices; pileipellis an ixotrichoderm with hyphae highly branched, coralloid with densely spinulose clavate, broadly clavate to globose terminal cells; fusoid to fusoid ventricose marginal cells with sparse spinulae with long narrow apices; fusoid to lanceolate, simple nonspinulose caulocystidia; habitat on dead, decaying bamboo and luminescent pileus and lamellae. The Indian collection matches closely with the description of the topotype (Desjardin et al. 2010) and also the description of the Malaysian material (Chew et al. 2014). However, pellucid striate pileus with thick gluten cover, slightly larger basidiospores, presence of two types of basidia, lack of nitrous or chlorine odor and exclusive habitat on dead decaying bamboo culms are the differences noticed in the present Indian collection.

A BLASTn search in GenBank using nrITS sequence of the present material showed $98.44 \%$ identity and $99 \%$ query cover with $M$. chlorophos (AB512312). The ML analysis shows 98\% bootstrap support with M. chlorophos (AB512312) from Japan (Fig. 3). Therefore, the differences noticed in the macro and micromorphological features of the Indian material may be considered as additional features.

Mycena chlorophos was initially described from Bonin Islands in 1860, Japan and later reported from both Old World and New World and hence currently considered as pantropical in distribution (Desjardin et al. 2010). Thus, the report of M. chlorophos from India is significant phytogeographically. 

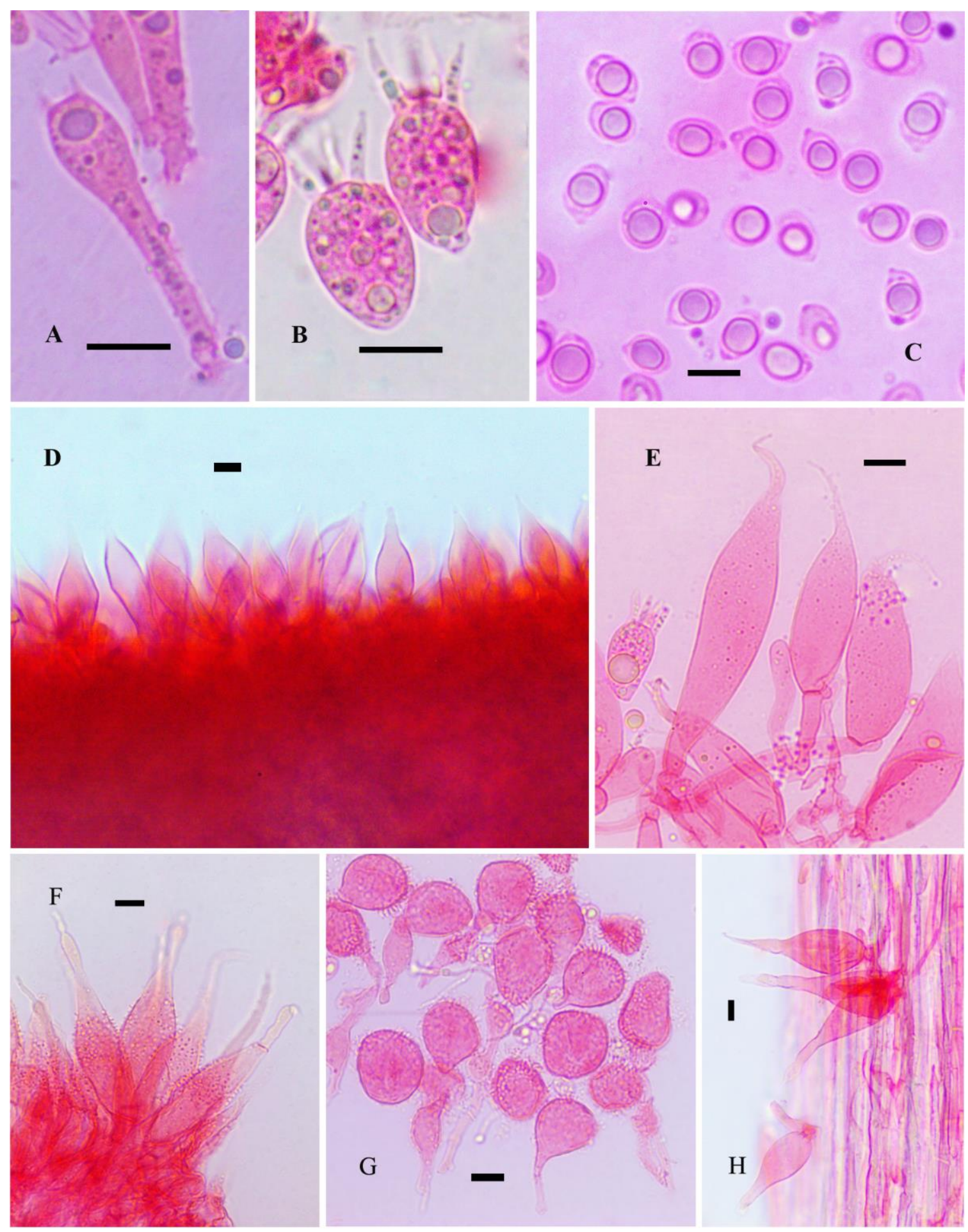

Fig. 2 - A Normal basidium. B Short basidia. C Basidiospores. D Lamella edge with cheilocystidia. E Cheilocystidia. F Pileus marginal cells. G Pileipellis terminal cells. H Caulocystidia. Scale bars: $\mathrm{A}-\mathrm{H}=10 \mu \mathrm{m}$

The Indian material is also similar to M. margarita (Murrill) Murrill and M. discobasis Métrod in gross morphology and in some microscopic characters. However, M. margarita can be separated by its smaller basidiomata (10-18 mm), smaller basidiospores (mean 6.9-4.4 $\mu \mathrm{m}$ ), strong chlorine odor and habitat on rotten wood. Smaller, dry to subviscid, minutely pruinose pileus (7-12 $\mathrm{mm})$, larger broadly ellipsoid basidiospores $(8.5-11 \times 6-7.5 \mu \mathrm{m})$, broadly clavate ventricose smaller cheilocystidia and habitat on rotten wood distinguishes $M$. discobasis from the present material. 
The only report of luminescent Mycena from India is that of Mycena deeptha Aravind. \& Manim. from Kerala State (Aravindakshan et al. 2012). However, M. deeptha is distinct by thin delicate basidiomes $(1-9.5 \mathrm{~mm})$, yellowish grey to brownish pruinose, plicato-sulcate pileus, smaller basidiospores $(7.6-3.8 \mu \mathrm{m})$, small vesiculose cheilocystidia and habitat on woody shells of fruits and decaying leaves. Morphological characteristics such as medium sized basidiomata with a discoid stipe base, gelatinous pileipellis composed of elements with thorn-like excrescences, nongelatinised stipitipellis and amyloid basidiospores place $M$. chlorophos in sect. Exornatae.

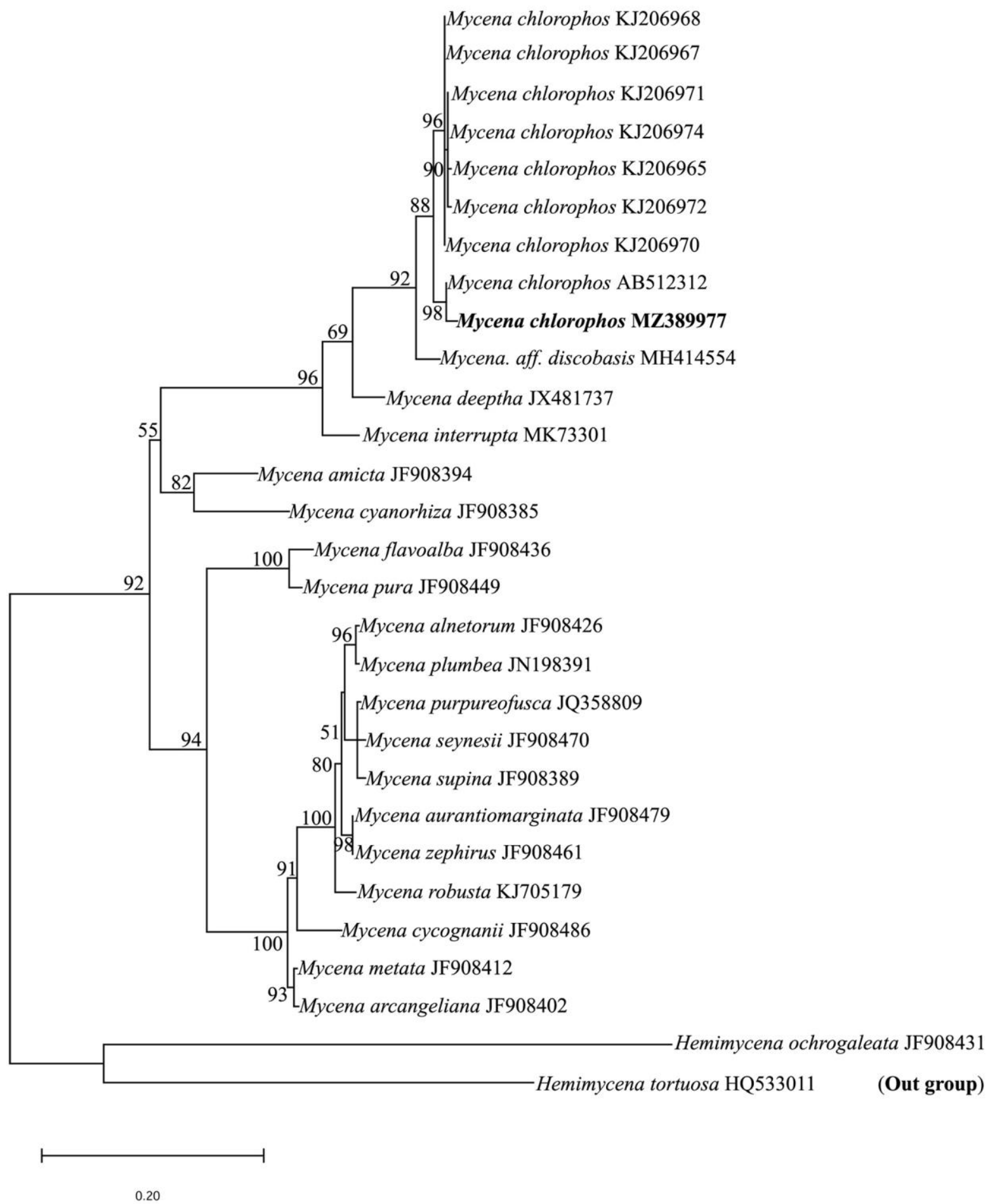

Fig. 3 - Maximum likelihood tree generated from ITS sequence data. Bootstrap values are indicated above/below branches. BS values greater than $50 \%$ are shown. Collection from Kerala, India is given in bold. GenBank accession numbers are given after the name of each taxon. 


\section{Acknowledgements}

The authors are thankful to the Director, JNTBGRI for facilities and Arya C.P. acknowledges financial support from the CSIR, India in the form of Junior Research Fellowship (File No: 09/592(0031)/2019-EMR-I).

\section{References}

Aravindakshan DM, Kumar TKA, Manimohan P. 2012 - A new bioluminescent species of Mycena sect. Exornatae from Kerala state, India. Mycosphere 3(5), 556-561. Doi 10.5943/mycosphere/3/5/4

Chang CC, Chi-Yu C, Wen-Wen LIN, Kao HW. 2020 - Mycena jingyinga, Mycena luguensis, and Mycena venus: Three new species of bioluminescent fungi from Taiwan. Taiwania 65(3), 396-406. Doi 10.6165/tai.2020.65.396

Chew ALC, Desjardin DE, Tan YS, Musa MY, Sabaratnam V. 2014 - Bioluminescent fungi from Peninsular Malaysia. Fungal diversity 70, 149-187. Doi 10.1007/s13225-014-0302-9

Cortés-Pérez A, Desjardin DE, Perry BA, Ramírez-Cruz V et al. 2019 - New species and records of bioluminescent Mycena from Mexico. Mycologia 111(2), 319-338.

Doi 10.1080/00275514.2018.1554172

Desjardin DE, Oliveira AG, Stevani CV. 2008 - Fungi bioluminescence revisited. Photochemical and Photobiological Sciences 7 (2), 170-182. Doi 10.1039/b713328f

Desjardin DE, Perry BA, Lodge DJ, Stevani CV, Nagasawa E. 2010 - Luminescent Mycena: new and noteworthy species. Mycologia 102(2), 459-477. Doi 10.3852/09-197

Hall TA. 1999 - Bio Edit: a user-friendly biological sequence alignment editor and analysis program for Windows 95/98/NT. Nucleic Acids Symposium Series 41, 95-98. Doi 10.11646/phytotaxa.372.1.5

Herring PJ. 1987 - Systematic distribution of bioluminescence in living organisms, Journal of Bioluminescence and Chemiluminescence 1, 147-163.

Izumitsu K, Hatoh K, Sumita T, Kitade Y et al. 2012 - Rapid and simple preparation of mushroom DNA directly from colonies and fruiting bodies for PCR. Mycoscience 53: 396-401.

Doi 10.1007/S10267-012-0182-3

Jayasiri SC, Hyde KD, Ariyawansa HA, Bhat J et al. 2015 - The Faces of Fungi database: fungal names linked with morphology, phylogeny and human impacts. Fungal Diversity 74(1), 3-18. Doi 10.1007/s13225-015-0351-8

Karunarathna SC, Mortimer PE, Tibpromma S, Dutta AK et al. 2020 - Roridomyces phyllostachydis (Agaricales, Mycenaceae), a new bioluminescent fungus from Northeast India. Phytotaxa 459 (2), 155-167. Doi 10.11646/phytotaxa.459.2.6

Kornerup A, Wanscher JH. 1978 - Methuen handbook of color. 3rd ed. London, Methuen. 252 p.

Kumar S, Stecher G, Li M, Knyaz C, Tamura K. 2018a - MEGAX: Molecular Evolutionary Genetics Analysis across Computing Platforms. Molecular Biology and Evolution 35(6), 1547-1549. Doi 10.1093/molbev/msy096

Kumar A, Vrinda KB, Pradeep CK. 2018b - Two new species of Crepidotus (Basidiomycota, Agaricales) from peninsular India. Phytotaxa 372 (1), 67-78

Kumar M, Kaviyarasan V. 2012 - Few common poisonous mushrooms of Kolli Hills, South India. Journal of Academia and Industrial Research 1 (1), 19-22.

Trifinopoulos J, Nguyen LT, Haeseler A, Minh BQ. 2016 - W-IQ-TREE: a fast-online phylogenetic tool for maximum likelihood analysis. Nucleic Acids Research 44 (W1), W232W235. Doi 10.1093/nar/gkw256

Vrinda KB, Pradeep CK, Abraham TK. 1999 - Bioluminescent agarics from Western Ghats. Mushroom Research 8 (2), 31-33.

White TJ, Bruns T, Lee SJWT, Taylor J. 1990 - Amplification and direct sequencing of fungal ribosomal RNA genes for phylogenetics. PCR protocols: a guide to methods and applications 18(1), 315-322. Doi 10.1016/b978-0-12-372180-8.50042-1 